\title{
Delayed pericardial effusion after left atrial appendage closure with the LAmbre device: importance of a fully open umbrella
}

\author{
Fangyi Xiao ${ }^{1}$, yanyan chen $^{2}$, yi he chen $^{3}$, Xiaodong Zhou ${ }^{4}$, Xinlei $\mathrm{Wu}^{4}$, xiao chen ${ }^{4}$, \\ Liangguo Wang ${ }^{5}$, Ying Fang ${ }^{2}$, Lan $\mathrm{Su}^{4}$, and weijian huang ${ }^{6}$ \\ ${ }^{1}$ the First Affiliated Hospital of Wenzhou Medical University ZheJiang 325000 P.R. China \\ ${ }^{2}$ The First Afliated Hospital of Wenzhou Medical University \\ ${ }^{3}$ the second afliated hospital of Wenzhou Medical University \\ ${ }^{4}$ The First Affiliated Hospital of Wenzhou Medical University \\ ${ }^{5}$ he First Affiliated Hospital of Wenzhou Medical University \\ ${ }^{6}$ The First Affiliated Hospital of Wenzhou Medical College
}

February 1, 2021

\begin{abstract}
Introduction: We aimed to investigate whether a modified implantation method facilitating a fully open umbrella can reduce the pericardial effusion/pericardial tamponade (PE/PT) rate after left atrial appendage closure (LAAC) with the LAmbre device compared with the conventional method $(\mathrm{CM})$ in patients with non-valvular atrial fibrillation (NVAF). Methods and results: Patients with NVAF who received either isolated LAAC or combined catheter ablation and LAAC using the LAmbre device at the First Affiliated Hospital of Wenzhou Medical University from January 2018 to December 2019 were enrolled. CM was used for device implantation in the initial 59 patients, while a modified method (MM) was used in the remaining 165 patients. Successful implantation was achieved in $98.3 \%$ of patients in the CM group and in $98.8 \%$ in the MM group. A higher rate of a fully open umbrella ( $98.8 \%$ vs $69 \%, \mathrm{P}<0.001)$, less requirement for recapture $(46 \%$ vs $62.1 \%, \mathrm{P}=0.036)$, and a lower incidence of delayed PE/PT (1.2\% vs $8.6 \%, \mathrm{P}=0.005)$ were found in the MM group compared with in the CM group. All of the five delayed PT events occurred in patients with combined treatment. An umbrella that was not fully open was the only factor associated with delayed PE/PT events in a multivariable Cox model. Conclusions: LAAC with the LAmbre device using an MM significantly increases the rate of a fully open umbrella and decreases the requirement for recapture and the incidence of delayed PE/PT. This method is more effective in patients with combined treatment.
\end{abstract}

\section{Introduction}

Transcatheter left atrial appendage closure (LAAC) has emerged as a reasonable alternative to long-term oral anticoagulant therapy (OAT) for stroke prevention in patients with non-valvular atrial fibrillation (NVAF). Randomized trials for the Watchman device ${ }^{1-3}$ and large, all-comer registries for Amplatzer devices ${ }^{4,5}$ have shown promising clinical outcomes in preventing stroke and avoiding bleeding events. Additionally, combined catheter ablation, including radiofrequency ablation and cryoablation with LAAC using Watchman or Amplatzer devices in a single procedure, were reported to be effective and safe strategies in patients who had indications for atrial fibrillation $(\mathrm{AF})$ ablation and $\mathrm{LAAC}^{6-9}$.

The LAmbre ${ }^{\mathrm{TM}}$ occluder was recently introduced to clinical practice and small registry studies have shown high success rates for implantation and acceptable periprocedural complication rates ${ }^{10-12}$. Data of a combined procedure with the LAmbre device are still lacking. Different to Amplatzer devices, the umbrella of the LAmbre device has a double stabilization design with eight small distal hooks that engage into the wall of the left atrial appendage (LAA) and eight U-shaped ends trapping in LAA trabeculations (Figure 1). 
Previous studies ${ }^{11,13}$ showed that delayed cardiac effusion/cardiac tamponade (PE/PT) events might occur following LAAC with the LAmbre device.

We retrospectively investigated the safety and short-term outcome in patients who underwent isolated LAAC with the LAmbre device or combined treatment of catheter ablation for AF and LAAC. A modified method $(\mathrm{MM})$ for LAmbre device implantation was compared with the conventional method (CM) in this study.

\section{Study population}

Patients with NVAF at a high risk of stroke who underwent LAmbre implants (Lifetech Scientific Corp., Shenzhen, China) at The First Affiliated Hospital of Wenzhou Medical University from January 2018 to December 2019 were included. The inclusion criteria for LAAC were as follows: age $>18$ years, $\mathrm{CHA}_{2} \mathrm{DS}_{2}$-VASc score [?]2, contraindication to long-term OAT, patients with bleeding events with or without anticoagulation therapy, stroke despite OAT, and intolerance or refusal to take OAT. The exclusion criteria were as follows: comorbid unstable angina or acute myocardial infarction, a left atrial inner diameter $>65 \mathrm{~mm}$, valvular heart disease, left atrial thrombus, a history of LAA ligation, a history of inferior vena cava filter placement surgery, severe infection, tumours, other diseases requiring systemic anticoagulation, new onset stroke events within 1 month, active bleeding disease, and a left ejection fraction [?]30\%. The conventional implantation method was used in the LAAC procedure in the initial 59 patients (CM group) and an MM was used in the subsequent 165 patients (MM group). All patients received pre-procedural transesophageal echocardiography (TEE) to assess anatomy of the LAA and to exclude thrombus of the LAA. Written informed consent was obtained from all of the participants. The study was approved by the ethics committee of the local institution.

\section{LAA closure with the LAmbre device}

The LAAC procedure was performed under local anaesthesia. Transseptal puncture was performed via right femoral venous access under fluoroscopic guidance. The sizes of the landing zone and the orifice were measured from LAA angiography at the right anterior oblique and caudal projection. The appropriate LAmbre size was selected on the basis of the intraoperative angiographic and preoperative TEE measurements. The size of the implant was 4 to $8 \mathrm{~mm}$ larger than the measured LAA orifice based on the clinical judgement of the operators. The CM was described as previously reported ${ }^{14}$. In brief, the delivery sheath was placed in the proximal part of the LAA. The distal umbrella was released into the LAA by stepwise pushing out of the device from the delivery sheath. Subsequently, the sheath was withdrawn to expose the proximal cover. The umbrella was adjusted to open as fully as possible. In the MM, the distal umbrella was initially half deployed outside of the LAA (defined as when a radio-opaque marker of the LAmbre device was located between two distal marker bands at the end of the delivery sheath and it was closer to the proximal marker band) and then gently "en-bloc" advanced into the proximal part of the LAA. Complete deployment of the umbrella to the landing zone was subsequently achieved by pushing out the device from the delivery sheath (Figure 2). The manipulation to expose the cover was the same as that for the CM. A fully open umbrella was defined as when the nadir of the U-shaped hooks was at the same level as the radio-opaque marker connecting the umbrella and cover in fluoroscopic imaging (Figure 1B). Stability was confirmed by the tug test. The position of the device and any presence of peri-device leakage were assessed by fluoroscopy and two-dimensional TEE $\left(0^{\circ}, 45^{\circ}, 90^{\circ}\right.$, and $\left.135^{\circ}\right)$ and following three-dimensional TEE under local laryngeal anaesthesia with tetracaine. Patients who could not tolerate a TEE examination were sedated with etomidate and fentanyl. Recapture and/or resizing of the device was attempted unless the device was in a satisfactory position and no major peri-device leakage (leakage [?]3 $\mathrm{mm}$ ) was achieved. The activated clotting time was adjusted to maintain 250 to $350 \mathrm{~s}$ during the procedure.

\section{Radiofrequency ablation for AF}

In patients with the combined procedure, radiofrequency ablation was performed before LAAC under conscious sedation, with fentanyl and midazolam for pain relief. Following double transseptal punctures, a left atrial (LA) anatomical map was created using a 10-pole spiral catheter (Lasso NAV; Biosense Webster, Inc., Diamond Bar, CA) under the guidance of a three-dimensional electroanatomical mapping system (CARTO 
3; Biosense Webster, Inc.). A contact-force catheter (Thermocool SmartTouch; Biosense Webster, Inc.) was used to perform ablation index-guided circumferential pulmonary vein isolation with target values (450 for the anterior wall and 350 for the posterior wall). The endpoint of ablation was bidirectional conduction block between the left atrium and pulmonary veins. In patients with persistent AF, cardioversions were delivered to restore sinus rhythm after circumferential pulmonary vein isolation. Substrate modification was then performed as previously described ${ }^{15}$.

\section{Post-procedural management and follow-up}

All patients received transthoracic echocardiography to rule out pericardial effusion on the following day of procedures. OAT was administrated for at least 45 days in patients with isolated LAAC and for 2 months in patients with the combined procedure. This treatment was followed by aspirin and clopidogrel for 6 months and aspirin or clopidogrel for life. At 45 days, 6 months, and 12 months of follow-up, TEE was performed to evaluate the position of the device, residual flow, and thrombus formation on the surface of the device.

\section{Major adverse events}

Major adverse events were defined as death, stroke, systemic embolism, device embolization, pericardial bleeding requiring an intervention (pericardial tamponade), atrio-oesophageal fistula, pulmonary vein stenosis, or other major bleeding requiring invasive treatment or blood transfusion.

\section{Statistical analysis}

Quantitative data are expressed as mean +- standard deviation and were compared using the two-tailed t test. Categorical data are expressed as percentages and were compared using the chi-square test. Comparisons of events by groups were made by Kaplan-Meier analysis and the log-rank test. Predictors of delayed PE/PT were analysed by univariate and subsequent multivariate Cox regression analyses. The results were expressed as hazard ratios (HRs) with $95 \%$ confidence intervals (CIs). $\mathrm{P}<0.05$ was considered statistically significant. All statistical analyses were performed using SPSS version 23.0.

\section{Results}

\section{Baseline characteristics of the patients}

Table 1 showed the baseline demographic data of the patients. The mean age of the patients was 71 years in the CM group and 70 years in the MM group. The prevalence of paroxysmal atrial fibrillation (PAF) was $55.6 \%$ and $50.0 \%$ in the $\mathrm{CM}$ and $\mathrm{MM}$ groups, respectively. The $\mathrm{CHA}_{2} \mathrm{DS}_{2}$-VASc scores were similar in the two groups. The mean HAS-BLED score was also similar in the two groups. There were significantly more patients with the combined procedure $(67.9 \%$ vs $45.8 \%, \mathrm{P}=0.003)$ and with prior stroke $(65.5 \%$ vs $50.8 \%$, $\mathrm{P}=0.048)$ in the MM group than in the CM group.

\section{Procedural characteristics}

The LAAC procedure with the LAmbre device was successfully performed in 58 (98.3\%) patients in the CM group and in $163(98.8 \%)$ patients in the MM group. There were no significant differences in LAA size and LAA anatomy between the two groups. The mean umbrella and cover diameters of the implanted device were similar in the two groups. The rate of a fully open umbrella was significantly higher in the MM group than in the $\mathrm{CM}$ group $(\mathrm{P}<0.001)$. The rate of a device requiring recapture and the mean number of recaptures were significantly lower in the MM group than in the CM group (both $\mathrm{P}<0.05$ ). LAAC with complete coverage of the $\mathrm{LAA}^{16}$ was achieved in most of the patients in both groups $(70.2 \%$ vs $60.3 \%$ ). The rates of partial prolapse and complete prolapse into the LAA neck were also similar in the two groups (Table 2).

\section{Adverse events}

No severe intra-procedural complications, including PE/PT, device embolization, air embolism, thromboembolism, and death, occurred in either group. Delayed PE/PT developed in five patients in the CM group and in two patients in the MM group (Table 3). Umbrellas that were not fully open were found in all of 
the five patients with delayed $\mathrm{PE} / \mathrm{PT}$ in the $\mathrm{CM}$ group, while fully open umbrellas were found in the two patients with delayed $\mathrm{PE} / \mathrm{PT}$ in the MM group (Figure 3). In the CM group, moderate PE was found by echocardiography in the $9^{\text {th }}$ patient with persistent $\mathrm{AF}$ on day 15 who underwent isolated LAAC and in the $52^{\text {nd }}$ patient with paroxysmal $\mathrm{AF}$ who received combined treatment 2 days after the procedure. Both of these patents were managed conservatively without further complications. Delayed PT occurred in three patients who received the combined procedure requiring intervention in the CM group. Sero-haemorrhagic liquid (haemoglobin value was close to that in blood) was drained in all of these patients. The $31^{\text {st }}$ patient with PAF developed PT on day 8 after the operation. She was successfully treated with pericardiocentesis with no long-term sequelae. The $42^{\text {nd }}$ patient with persistent AF developed PT on day 18 after the procedure and was treated with pericardiocentesis. The $59^{\text {th }}$ patient with PAF suffered from progressive dyspnoea from 8 $\mathrm{h}$ after the procedure in the night and received pericardiocentesis $15 \mathrm{~h}$ after the procedure. Cardiac arrest occurred in preparation of pericardiocentesis. The patient died 7 days after the procedure owing to multiorgan failure. In the MM group, the $110^{\text {th }}$ patient with persistent $\mathrm{AF}$ who received the combined procedure developed PT on day 51 after the procedure and was successfully treated with pericardiocentesis. The $174^{\text {th }}$ patient with PAF who underwent the combined procedure developed PT 30 min after the procedure. Pericardiocentesis was performed immediately in this patient. Surgical management of PT was required because blood continually flowed out through the pigtail catheter. A small branch of the left circumflex artery was bleeding, which was likely perforated by a small shoulder hook of the device, was explored during surgery. Excision of the LAA and coronary artery suture were performed. The patient was discharged in a good condition on day 20 after surgery.

The delayed PE/PT rate was significantly lower in the MM group than in the CM group (8.6\% vs $1.2 \%$, $\log$-rank $\mathrm{P}=0.005)$. An umbrella that was not fully open was the only factor associated with delayed $\mathrm{PE} / \mathrm{PT}$ events in univariate analysis (HR: 0.038, 95\% CI: 0.007-0.194; $\mathrm{P}<0.001)$ and in the multivariable Cox model (HR: 0.026, 95\% CI 0.004-0.183; $\mathrm{P}<0.001$ ) after adjusting for age, sex, type of AF, type of procedure, HAS-BLED score, type of anticoagulants, mean number of recaptures, device position, LAA anatomy, the umbrella diameter divided by the land zone diameter (measured at RAO 30deg, CAU 20deg projection) ratio and the cover diameter divided by the orifice diameter (measured at RAO 30deg, CAU 20deg projection) ratio (Tables 4,5$)$.

The $68^{\text {th }}$ patient had an ischaemic stroke 2 days after the procedure. Severe stenosis of the basilar artery was found as the cause of the stroke event. The $114^{\text {th }}$ patient with the combined procedure suffered from gastrointestinal bleeding 24 days after the operation owing to an intestinal tumour. Minor complications included a haematoma at the puncture site in one patient with isolated LAAC in the CM group and in two patients with the combined procedure in the MM group, and a pseudoaneurysm at the puncture site in one patient with combined treatment in the MM group. No device embolization, device-related thrombus, stroke, death, atrio-oesophageal fistula, or pulmonary vein stenosis was observed during 3 months of follow-up.

\section{Short-term TEE follow-up}

The first TEE follow-up rates were similar in both groups ( $87.9 \%$ vs $87.7 \%, \mathrm{P}=0.968)$. The complete seal rates in the $\mathrm{CM}$ and $\mathrm{MM}$ groups were $68.6 \%$ and $65.0 \%$, respectively. Peri-device leakage [?]3 $\mathrm{mm}$ was observed in the majority of patients during follow-up TEE examinations, except for two patients who had peri-device leakage of $4 \mathrm{~mm}$ in the MM group (Figure 4).

\section{Discussion}

To the best of our knowledge, this is the largest study of LAAC using the LAmbre device. We found a relatively high incidence of delayed PE/PT after LAAC with the LAmbre device using a conventional implantation method, mainly in patients with combined treatment. The MM for implantation of the LAmbre device significantly facilitated full opening of the umbrella, reduced the requirement of the recapture manoeuver, and decreased the incidence of $\mathrm{PE} / \mathrm{PT}$. In multivariate Cox regression analysis, an umbrella that was not fully open was the only factor independently associated with delayed PE/PT events.

$\mathrm{PE} / \mathrm{PT}$ is a serious complication, which mostly occurs during LAAC, but rarely occurs after this procedure ${ }^{17}$. 
Schmidt et $\mathrm{al}^{18}$ found that the PE/PT rate was significantly higher in patients who were implanted with the Watchman device in the EWOLUTION study during sinus rhythm than in AF rhythm $(0.2 \%$ vs $1.5 \%$; $\mathrm{P}=0.02)$ at day 30 post-implantation. Delayed PT events that required intervention only occurred in LAAC during sinus rhythm. The investigators speculated that the main mechanism for their finding was LAA contraction during sinus rhythm exerting mechanical force on the device, which eventually led to PE/PT. Wolfrum et al ${ }^{19}$ compared the efficacy and safety of LAAC with the Amplatzer Cardiac Plug according to the different position of the device disc. No evidence for a difference in the occurrence PE/PT was found between patients with complete versus incomplete Amplatzer Cardiac Plug disc coverage of the LAA ostium. In contrast, a recent study ${ }^{16}$ on the Amulet device reported a tendency toward more $\mathrm{PE} / \mathrm{PT}$ events after LAAC with incomplete coverage of the LAA ostium by the proximal Amulet disc $(\mathrm{P}=0.07)$. This previous study suggested that suboptimal positioning of the device might be associated with worse periprocedural outcomes independent of repositioning or exchange of the device during the procedure. In our study, delayed $\mathrm{PE} / \mathrm{PT}$ events were neither associated with the coverage position nor recapture operation as analysed by univariate and subsequent multivariate Cox regression analyses.

Huang et $\mathrm{a}^{10}$ reported encouraging clinical outcomes in patients with NVAF who had implantation of the LAmbre LAA occluder. Three PT events (timing of the events was not specified) were documented in their study. Park et al ${ }^{11}$ reported 2/60 (3.3\%) patients who underwent LAAC with the LAmbre device and suffered from PT at days 8 and 33 after the procedure. Pericardiocentesis was required for these two patients. A recent study with a small sample size ${ }^{13}$ showed that $5(29.4 \%)$ delayed PE events occurred in 17 patients who underwent LAAC with the LAmbre device during 3 months of follow-up. Data from LAAC with the LAmbre device are relatively inadequate and the safety of combining LAAC with the LAmbre device and catheter ablation is unknown. In the present study, five of six patients with delayed $\mathrm{PE} / \mathrm{PT}$ required intervention in those with the combined procedure. Only one PE event occurred among patients with non-PAF who underwent isolated LAAC.

The umbrella of the LAmbre device has double hooks (eight small distal hooks engaging into the LAA wall and eight U-shaped large hooks trapping in LAA trabeculations) with the purpose of decreasing the risk of device embolization. However, these eight U-shaped large hooks are bare and slender. A narrow space with widespread trabeculations in the LAA frequently interferes with opening of the umbrella. A stronger mechanical force against the LAA wall might develop when any of these hooks are not fully opened. Therefore, full opening of the umbrella is important and is considered as one of the COST criteria (including umbrella deployed beyond the Circumflex artery; umbrella fully Open; peri-device with optimal Sealing (leakage [?] $3 \mathrm{~mm}$ ) for release of the LAmbre device; and device stability confirmed by the Tug test) for release of the LAmbre device. The modified implantation method in which the umbrella is initially deployed half open outside of the LAA and complete deployment into the landing zone facilitates full opening of the umbrella. In our study, all of the patients with delayed PE/PT showed incomplete opening of the umbrella in fluoroscopic imaging in the CM group. Additionally, incomplete opening of the umbrella was significantly associated with occurrence of delayed PE/PT events as shown in the univariate model and multivariate Cox regression analysis. The nadir of the U-shaped hooks at the same level as the radio-opaque marker connecting the umbrella and the cover as shown in fluoroscopic imaging is an important sign of full opening of the umbrella. However, this is still insufficient to guarantee an appropriate mechanical force from the double hooks against the LAA wall because full opening of the umbrella was found in another two patients with delayed PT in the MM group. One of these patients required surgical management because a small branch of the left circumflex artery was likely injured by the small hooks. This case was considered as an exception. In patients with the combined procedure, contractile function of the LAA was gradually restored when sinus rhythm was maintained after the procedure. We speculate that relative movement between the bare large hooks and the LAA wall causing friction during sinus rhythm was the leading cause of perforation. Therefore, we strongly recommend using this MM for patients with the combined procedure or isolated LAAC in those with PAF. Performance of routine echocardiographic follow-up in this patient population may be necessary. The manufacturer of the LAmbre device might manage with the eight U-shaped big hooks in the next-generation product design. 


\section{Limitations}

The retrospective, nonrandomized, single-centre design of our study has some limitations. Because the CM group comprised the initial patients who underwent LAAC, there might have been a learning curve, which would be in favour of the MM group. However, PT events occurred in the $110^{\text {th }}$ and $174^{\text {th }}$ patients in the MM group. This finding suggests there was no significant correlation between the incidence of PE/PT and the learning curve. Additionally, the limited number of events limited the power for our ability to detect a significant association, as shown by the wide confidence intervals.

\section{Conclusions}

The conventional implantation method for the LAmbre device might be associated with a high incidence of delayed $\mathrm{PE} / \mathrm{PT}$ in patients with the combined procedure. The MM with the intention of reducing tension from the umbrella toward the LAA wall significantly improves the rate of full opening of the umbrella, avoids extensive recapture of the device, and reduces the incidence of delayed PE/PT events.

\section{Acknowledgements}

We thank Ellen Knapp, PhD, from Liwen Bianji, Edanz Group China (www.liwenbianji.cn/ac), for editing the English text of a draft of this manuscript.

\section{References}

1. Holmes DR, Jr., Kar S, Price MJ, et al. Prospective randomized evaluation of the Watchman Left Atrial Appendage Closure device in patients with atrial fibrillation versus long-term warfarin therapy: the PREVAIL trial. J Am Coll Cardiol. 2014;64(1):1-12.

2. Holmes DR, Reddy VY, Turi ZG, et al. Percutaneous closure of the left atrial appendage versus warfarin therapy for prevention of stroke in patients with atrial fibrillation: a randomised non-inferiority trial.Lancet. 2009;374(9689):534-542.

3. Reddy VY, Doshi SK, Kar S, et al. 5-Year Outcomes After Left Atrial Appendage Closure: From the PREVAIL and PROTECT AF Trials. J Am Coll Cardiol. 2017;70(24):2964-2975.

4. Landmesser U, Tondo C, Camm J, et al. Left atrial appendage occlusion with the AMPLATZER Amulet device: one-year follow-up from the prospective global Amulet observational registry.EuroIntervention. 2018;14(5):e590-e597.

5. Tzikas A, Shakir S, Gafoor S, et al. Left atrial appendage occlusion for stroke prevention in atrial fibrillation: multicentre experience with the AMPLATZER Cardiac Plug. EuroIntervention.2016;11(10):1170-1179.

6. Wintgens L, Romanov A, Phillips K, et al. Combined atrial fibrillation ablation and left atrial appendage closure: long-term follow-up from a large multicentre registry. Europace.2018;20(11):1783-1789.

7. Fassini G, Conti S, Moltrasio M, et al. Concomitant cryoballoon ablation and percutaneous closure of left atrial appendage in patients with atrial fibrillation. Europace. 2016;18(11):1705-1710.

8. Calvo N, Salterain N, Arguedas H, et al. Combined catheter ablation and left atrial appendage closure as a hybrid procedure for the treatment of atrial fibrillation. Europace.2015;17(10):1533-1540.

9. Phillips KP, Romanov A, Artemenko S, et al. Combining left atrial appendage closure and catheter ablation for atrial fibrillation: 2-year outcomes from a multinational registry. Europace.2020;22(2):225-231.

10. Huang H, Liu Y, Xu Y, et al. Percutaneous Left Atrial Appendage Closure With the LAmbre Device for Stroke Prevention in Atrial Fibrillation: A Prospective, Multicenter Clinical Study. JACC Cardiovasc Interv. 2017;10(21):2188-2194.

11. Park JW, Sievert H, Kleinecke C, et al. Left atrial appendage occlusion with lambre in atrial fibrillation: Initial European experience. Int J Cardiol. 2018;265:97-102. 
12. Schnupp S, Liu XX, Buffle E, et al. Late clinical outcomes of lambre versus amplatzer occluders for left atrial appendage closure. J Cardiovasc Electrophysiol. 2020;31(4):934-942.

13. Feng XF, Zhang PP, Sun J, Wang QS, Li YG. Feasibility and Safety of Left Atrial Appendage Closure Using the LAmbre Device in Patients with Nonvalvular Atrial Fibrillation with or Without Prior Catheter Ablation.Int Heart J. 2019;60(1):63-70.

14. Lam YY. A new left atrial appendage occluder (Lifetech LAmbre Device) for stroke prevention in atrial fibrillation. Cardiovasc Revasc Med. 2013;14(3):134-136.

15. Yang B, Jiang C, Lin Y, et al. STABLE-SR (Electrophysiological Substrate Ablation in the Left Atrium During Sinus Rhythm) for the Treatment of Nonparoxysmal Atrial Fibrillation: A Prospective, Multicenter Randomized Clinical Trial. Circ Arrhythm Electrophysiol. 2017;10(11).

16. Oraii Yazdani K, Mitomo S, Ruparelia N, et al. Percutaneous left atrial appendage occlusion with the Amulet device: The impact of device disc position upon periprocedural and long-term outcomes. Catheter Cardiovasc Interv. 2019;93(1):120-127.

17. Schroeter MR, Danner BC, Hunlich M, Schillinger W. Uncommon delayed and late complications after percutaneous left atrial appendage closure with Amplatzer $((\mathrm{R}))$ Cardiac Plug. Clin Res Cardiol.2014;103(4):285-290.

18. Schmidt B, Betts TR, Sievert H, et al. Incidence of pericardial effusion after left atrial appendage closure: The impact of underlying heart rhythm-Data from the EWOLUTION study. J Cardiovasc Electrophysiol. 2018;29(7):973-978.

19. Wolfrum M, Attinger-Toller A, Shakir S, et al. Percutaneous left atrial appendage occlusion: Effect of device positioning on outcome. Catheter Cardiovasc Interv. 2016;88(4):656-664.

Figure legends

Figure 1 Structural characteristics of the LAmbre device and umbrella with or without full opening in fluoroscopic imaging. A) Structural characteristics of the LAmbre device. B) The nadir of U-shaped hooks of the device at the same level as a radio-opaque marker connecting the umbrella and cover is a sign of full opening of the umbrella. C) The nadir of U-shaped hooks of the device at a different level to that of a radio-opaque marker is a sign of an umbrella without full opening.

Figure 2 Step by step illustration of implantation of the LAmbre device in the MM group.

A) The distal umbrella was initially half deployed outside of the LAA (defined as when a radio-opaque marker of the LAmbre device was located between two distal marker bands at the end of the delivery sheath and it was closer to the proximal marker band). B) Then it was gently "en-bloc" advanced into the proximal part of the LAA. C) Complete deployment of the umbrella to the landing zone was subsequently achieved by pushing out the device from the delivery sheath. D) The sheath was withdrawn to expose the proximal cover.

Figure 3 Different opening status of the umbrella after release in patients with delayed PE/PT. Opening status of the umbrella after release in patients with delayed $\mathrm{PE} / \mathrm{PT}$ in the $\mathrm{CM}$ group, including the $9^{\text {th }}$ patient $(\mathrm{A})$, the $31^{\text {st }}$ patient $(\mathrm{B})$, the $42^{\text {nd }}$ patient $(\mathrm{C})$, the $52^{\text {nd }}$ patient $(\mathrm{D})$, and the $59^{\text {th }}$ patient $(\mathrm{E})$. Opening status of the umbrella after release in patients with delayed PT in the MM group, including the $110^{\text {th }}$ patient $(\mathrm{F})$ and the $174^{\text {th }}$ patient $(\mathrm{G})$.

Figure 4 Residual flow at first TEE follow-up A) in total; B) in isolated LAAC; and C) in the combined procedure. 

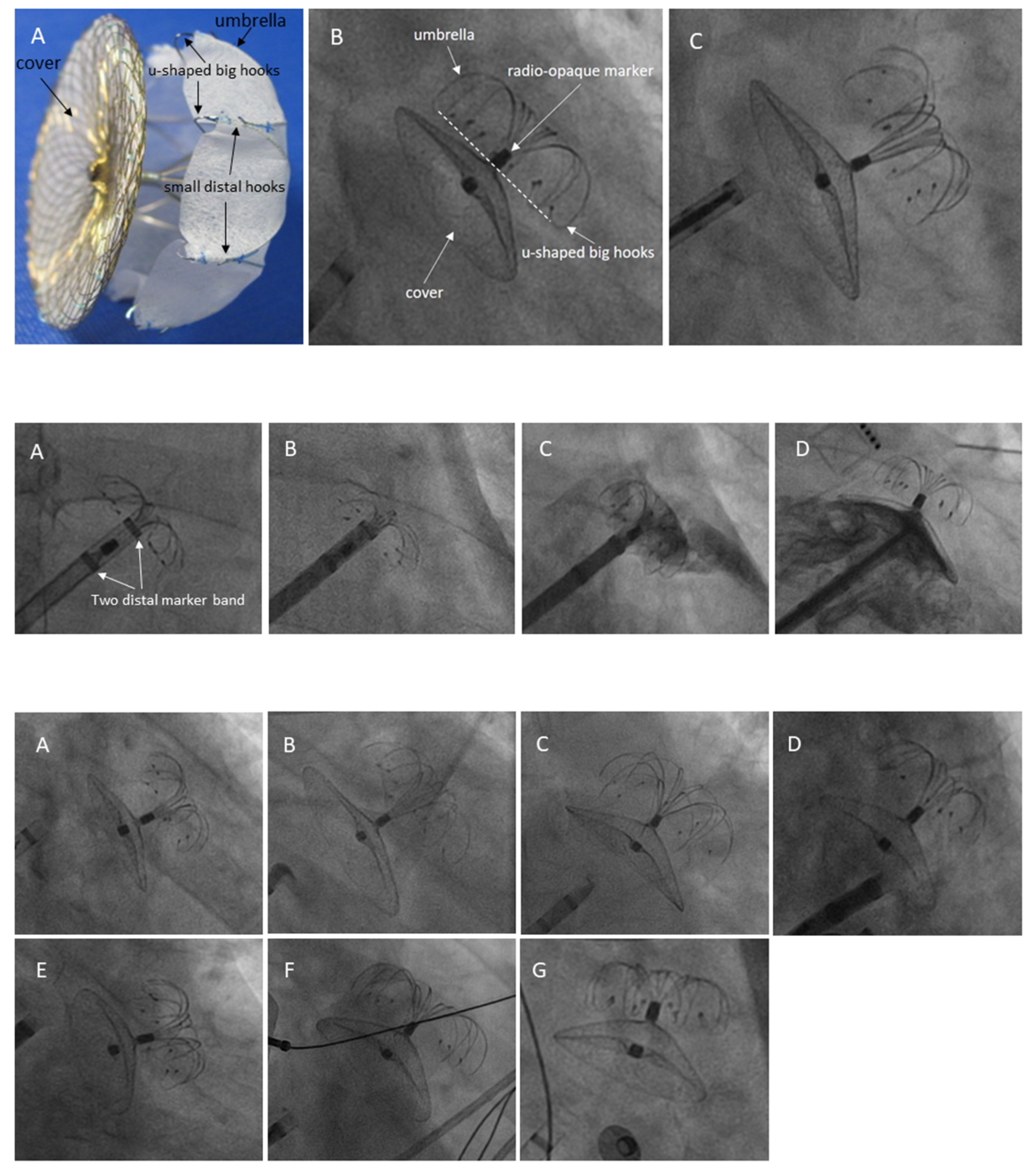

A

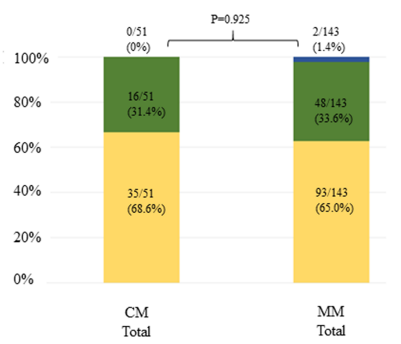

B

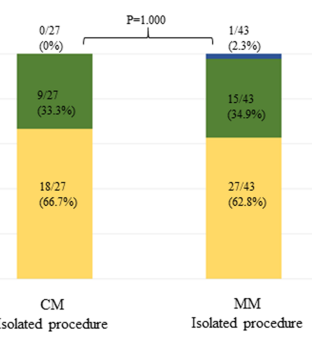

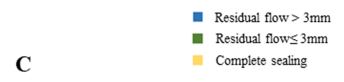

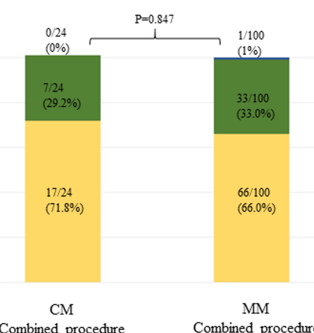

Hosted file

Table 1.pdf available at https://authorea.com/users/365858/articles/506828-delayedpericardial-effusion-after-left-atrial-appendage-closure-with-the-lambre-deviceimportance-of-a-fully-open-umbrella

\section{Hosted file}


Table 2.pdf available at https://authorea.com/users/365858/articles/506828-delayedpericardial-effusion-after-left-atrial-appendage-closure-with-the-lambre-deviceimportance-of-a-fully-open-umbrella

\section{Hosted file}

Table 3.pdf available at https://authorea.com/users/365858/articles/506828-delayedpericardial-effusion-after-left-atrial-appendage-closure-with-the-lambre-deviceimportance-of-a-fully-open-umbrella

\section{Hosted file}

Table 4.pdf available at https://authorea.com/users/365858/articles/506828-delayedpericardial-effusion-after-left-atrial-appendage-closure-with-the-lambre-deviceimportance-of-a-fully-open-umbrella

\section{Hosted file}

Table 5.pdf available at https://authorea.com/users/365858/articles/506828-delayedpericardial-effusion-after-left-atrial-appendage-closure-with-the-lambre-deviceimportance-of-a-fully-open-umbrella 